\title{
Abundance of Two Generalist Insect Pests of Cassava (Manihot esculenta Crantz) as Influenced by NPK Fertilization
}

\author{
James I. OKO ${ }^{1 *}$, Bonaventure C. ECHEZONA ${ }^{1}$, Christian U. AGBO ${ }^{1}$, \\ Stella O. MUOJIAMA ${ }^{2}$
}

\author{
${ }^{1}$ University of Nigeria, Department of Crop Science, Nsukka, Enugu State, \\ Nigeria; bonaventure.echezona@unn.edu.ng; christian.agbo@unn.edu.ng; jamesi4great@gmail.com (*correspondence author) \\ ${ }^{2}$ Nnamdi Azikiwe University, Department of Crop Science and Horticulture, Awka, Anambra, Nigeria; stellypotential@yahoo.com
}

\begin{abstract}
The influence of NPK fertilizer on the population of two generalist pests, grasshopper (Zonocerus variegatus) and termites (Isoptera spp.) on some cassava varieties (Manihot esculenta Crantz), was investigated in a field trial carried out at Teaching and Research Farm of Department of Crop science, University of Nigeria, Nsukka. The treatments comprised all possible combinations of four rates of fertilizer application $(0 \mathrm{~kg} / \mathrm{ha}, 200 \mathrm{~kg} / \mathrm{ha}, 400 \mathrm{~kg} / \mathrm{ha}$ and $600 \mathrm{~kg} / \mathrm{ha})$, four improved cassava varieties ('TMS 01-1368', 'TME 419', 'TMS 98-05-50', 'TMS $0510^{\circ}$ ') and three modes of fertilizer application (Single, Split and Split-split) arranged in a factorial experiment and laid out in a randomized complete block design (RCBD). There were three replications of each treatment. Data were collected on $Z$. variegatus and termite incidences. In each season, incidence of $Z$. variegatus on cassava showed significant $(\mathrm{p}<0.05)$ difference among varieties. Varieties 'TMS 01-1368', 'TMS 0510 ,' and 'TMS 98-05-05,' were significantly $(\mathrm{p}<0.05)$ more susceptible to the pest compared to variety 'TME 419'. Incidence of termites on cassava differed significantly $(\mathrm{p}<0.05)$ among varieties in the late season and variety TMS 0510 ' was more susceptible to this pest than other varieties. Rates of NPK fertilizer had significant $(\mathrm{p}<0.05)$ influence on $Z$. variegatus incidence only. Application of various NPK fertilizer rates increased the incidence of $Z$. variegatus on the varieties compared to plots without fertilizer $(0 \mathrm{~kg} / \mathrm{ha})$. The abundance of $Z$. variegatus in cassava field was influenced by fertilization and variety while termites were influenced by variety.
\end{abstract}

Keywords: Cassava; generalist pests; incidence; insect-plant interaction; NPK

\section{Introduction}

Soil fertility practices have serious influence on the physiological susceptibility of crops to insect. It can either affect the resistance of individual plant to attack or alter plant acceptability to certain herbivores. Application of nitrogen in form of urea to cotton plant enhanced the population build-up of whitefly while potassium fertilization improved host plant resistance to pests (Amtmann et al., 2008; Sati, 2012). Also adjudged to have low incidences of herbivores are plants growing in soils with optimum fertility while experiencing little or no stress. Its natural resistance is typically high and so is the incidence of predators (NCO, 2019). Although organic manure supplies nutrients to the soil, it improves the soil physical and chemical conditions and the yield of crops. It could lead to increase in pest numbers and damage caused to plants (Ikpe and Powell, 2002; Ewulo, 2005; Dauda et al., 2008; Ogedegbe et al., 2015; Baidoo et al., 2018). However, the compound fertilizer (NPK) has been seen to promote pest infestation in some situations while enhancing host plant resistance in others (Ogbalu, 1999; Yardim and Edwards, 2004). Even oviposition behaviour of bertha armyworm and larval performance was influenced by fertilizer treatment and canola variety, with moderate and high fertilizer treatments, giving similar number of eggs laid (Weedraddana and Evenden, 2018).

The behaviour of an arthropod as either generalist or specialist pest could be relevant in insect-plant interactions. Feller (1995) reported that the herbivory of dwarf red mangrove trees by two specialist herbivores of the tree increased with the application of P and NPK fertilizers. Fertilization had no effects on the feeding rates of generalist herbivores. Zonocerus variegatus (L), a tropical insect that belongs to the order Orthoptera and family Pyromorphidae, 
can be classified as a generalist pest of many crops including cassava. In Nigeria, it usually thrives on uncultivated land with the nymphs and adult stage sharing the same habitat. After cultivation, cassava (Manihot esculenta Crantz), is one of the major crops damaged in the south (Youdeowei, 1974; Page, 1978; Ademolu et al., 2011). $Z$. variegatus attacks in Africa are reported as causing severe crop damage, but reliable data on actual root yield loss is scarce. In the region, defoliation of several hectares of cassava has been linked to its outbreaks (Bellotti, 2002).

In southern Nigeria, it has been observed that abundance and population of grasshoppers could be determined or influenced by certain human activities that modify the environment including application of fertilizers and pesticides (Oku et al., 2011). It is not clear, however fertilizer rates that enhance infestation. Therefore, this study is aimed at determining the influence of cassava variety, rate and mode of NPK fertilization on the abundance of Zonocerus variegatus and termites in the cassava field.

\section{Materials and Methods}

The experiments were planted in May and July 2014 at the Teaching and Research farm of Department of Crop Science, University of Nigeria, Nsukka $\left(06^{\circ} 52^{\prime} \mathrm{N}, 07^{\circ} 24^{\prime} \mathrm{E}\right.$; $447.26 \mathrm{~m}$ above mean sea level). The soil is broadly characterized as sandy clay loam ultisol (oxic paleustult) and belongs to Nkpologu series (Mbagwu, 1992).

\section{Experimental materials}

Four improved cassava varieties were sourced from National Root Crop Research Institute (NRCRI), Umudike Umuahia, Abia state, Nigeria. Hardwood stem cuttings were collected from healthy plants and used as planting materials. The cassava varieties were: TMS 011368, TME 419, TMS 98-05-05 and TMS 0510 while NPK: 15:15:15 were sourced from Ogige market Nsukka was used.

\section{Land preparation and treatment application}

The field preparation for the first establishment commenced in May, 2014 while that of second site started in July, 2014 (accounting for early and late planting), with ploughing and harrowing of the field. The field was further demarcated into three blocks; individual blocks were later divided into 48 plots each. Both blocks and plots were separated by $1 \mathrm{~m}$ path way, each plot measuring $5 \mathrm{~m} \times 2 \mathrm{~m}$ to give $10 \mathrm{~m}^{2}$ per plot. Planting was done at a spacing of $1 \mathrm{~m}$ x $\operatorname{lm}(10,000$ plants/ha) using stem cuttings, 20 to $25 \mathrm{~cm}$ long. The treatments comprised all possible combinations of four rate of NPK fertilizer with three modes of application and four improved cassava varieties arranged in a factorial experiment and laid out in a randomized complete block design (RCBD) with three replications.

Factor A represented cassava varieties viz: TMS 011368, TME 419, TMS 98-05-05 and TMS 0510 while the four rates of NPK fertilizer representing Factor B included: $0 \mathrm{~kg} / \mathrm{ha}\left(0 \mathrm{~g} / 10 \mathrm{~m}^{2}\right), 200 \mathrm{~kg} / \mathrm{ha}\left(200 \mathrm{~g} / 10 \mathrm{~m}^{2}\right), 400 \mathrm{~kg} / \mathrm{ha}$ $\left(400 \mathrm{~g} / 10 \mathrm{~m}^{2}\right)$ and $600 \mathrm{~kg} / \mathrm{ha}\left(600 \mathrm{~g} / 10 \mathrm{~m}^{2}\right)$. The mode of fertilizer application representing Factor $C$ included the single applications ( 4 weeks after planting - WAP). Split applications (each single rate divided into two equal parts and applied at 4 and 8 weeks after planting). Split- split applications (each single dose divided into three equal parts and applied in three different periods at at 4, 8 and 12 weeks after planting).

Soil samples from the experimental sites were collected using soil auger sampler at $25-\mathrm{cm}$ soil depth before the commencement of the experiment. The samples were bulked together air-dried and a composite sample taken for physical and chemical analysis and these properties were determined: particle size distribution, chemical properties, exchangeable bases, cat ion exchange capacity (CEC) and exchangeable acidity. Cassava stems were kept under shade days after collection to avoid dehydration. NPK fertilizer corresponding to the appropriate treatment rates were applied in band method. Hoe weeding was carried out three times $(3,12$ and 24 weeks after planting (WAP)). To avoid undue interference with insect fauna, no insecticide was applied to the plants. Climatic data such as: monthly mean rainfall, temperature and relative humidity of the area were collected from the meteorological station of Department of Crop science, University of Nigeria for the period of the study (2014-2015).

\section{Data collection}

Percentage incidence of Zonocerus variegatus: $Z$. variegatus incidence was monitored starting from one (1) month after planting (MAP) to twelfth (12) month after planting (MAP). Zonocerus variegatus was collected using sweep net from each plant sampled. Each plant was checked thoroughly for the insect. Samples of Zonocerus spp. found were taken to the laboratory for counting. Sampling was done mainly in the relatively cool period of daylight, between 7.00 and 10:00 a.m., or between 4.00 and 6.00 p.m., when the insects are less active than at other times of the day. Zonocerus variegatus incidence was determined by dividing the total number of Zonocerus variegatus (ZV) infested plants by total number of plants, multiplied by one hundred, thus:

Percentage infested plants $=($ No. of plants infested with $\mathrm{ZV}$ per plot/total number of plants per plot $) \times 100$

Percentage incidence of termite: Termite damage was monitored starting one (1) MAP and was continued throughout the growing period, cassava cuttings damaged by termites were noted. Termite damage was determined by dividing the total number of termite-damaged plants (TDP) by total number of plants, multiplied by one hundred, thus:

Percentage infested plants $=$ No. of plants infested with TDP per plot/total number of plants per plot $\times 100$

\section{Statistical analysis of data}

All data collected on incidences of $Z$. variegatus and termite (insects count) were normalized using the square root transformation procedure before analyses of variance were carried out on them or their transformed values. Data were analysed using GENSTAT Discovery Edition 4 software. Separation of treatment means was done using Least Significant Difference (F-LSD) as outlined by Obi (2002). 


\section{Results}

\section{Meteorological data}

Results in Fig. 1 show the mean monthly rainfall, temperature and relative humidity during the period of the study (2014-2015).

Rainfall: considering the period of these experiments, rainfall was high with a better spread in 2014 compared to 2015. Although the rain stabilized earlier in 2015 compared to 2014, it was sustained for a couple of months in 2014 compared to 2015. Finally, the peak of rainfall (401.99$\mathrm{mm}$ ) in the period of the study was in September 2014 compared to highest rainfall (267.98-mm) in May 2015.
Temperature: generally, temperature (maximum and minimum) was higher in 2015 compared to 2014. In May 2014 both maximum and minimum temperatures were (28.29 and $21.06^{\circ} \mathrm{C}$ ) while that of May, 2015 were $(30.71$ and $21.81{ }^{\circ} \mathrm{C}$ ). Then in July 2014 both temperatures were $\left(27.74\right.$ and $201.81{ }^{\circ} \mathrm{C}$ ) while there was a slight increase in temperatures $\left(28.87\right.$ and $\left.20.61^{\circ} \mathrm{C}\right)$ of July 2015 .

Relative humidity: the data showed that the mean monthly humidity at 10:00 am and 4:00 pm for 2014 (72.2 $\%, 71.98 \%)$ were compared to that of 2015 (70.97\%, 69.3 $\%)$.

The variables tend to interact at various points from November 2014 to April 2015.

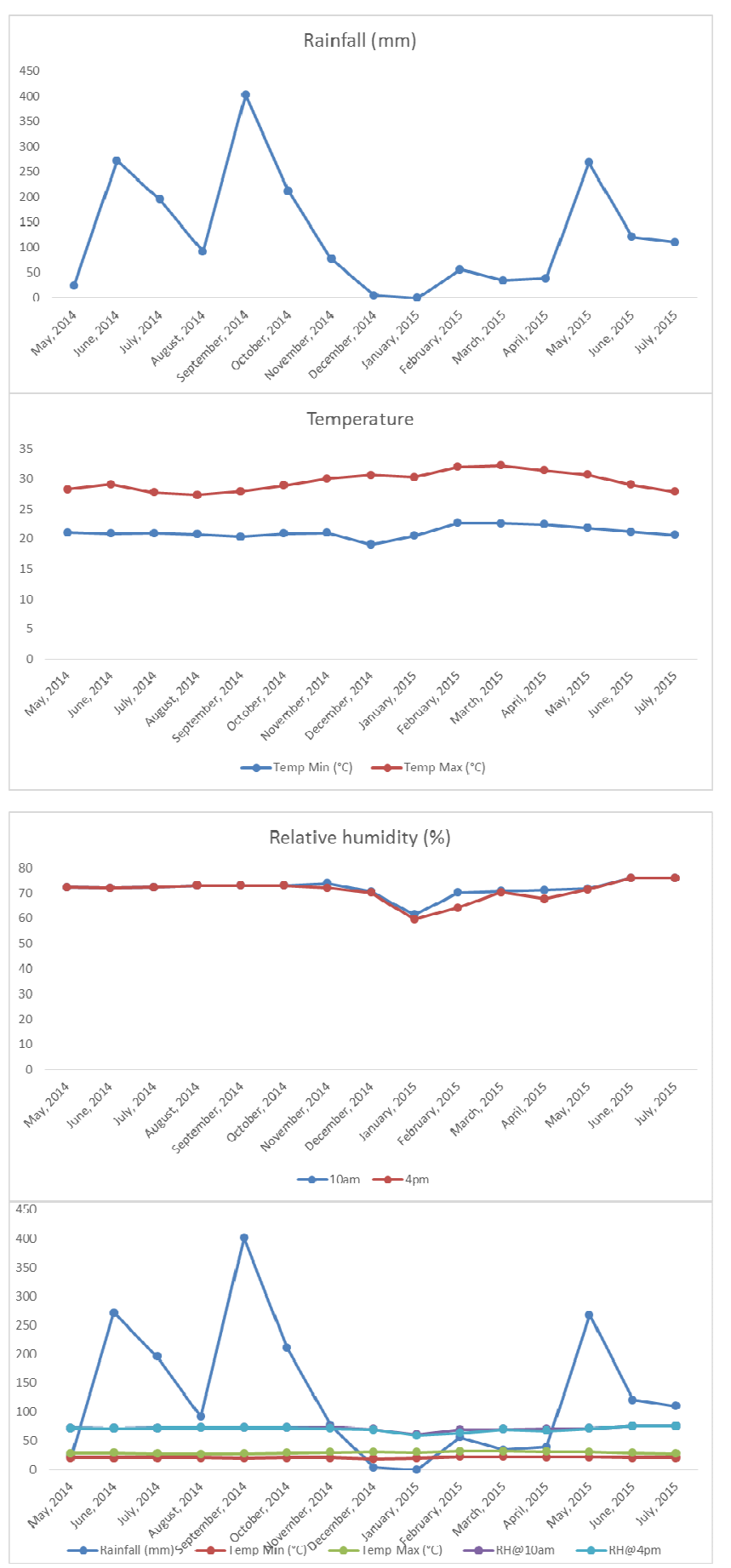

Fig. 1. Mean monthly rainfall $(\mathrm{mm})$, temperature $\left({ }^{\circ} \mathrm{C}\right)$, and relative humidity $(\%)$ during the period of study $(2014-2015)$ Source: Meteorological station, Department of Crop Science, University of Nigeria 
Physiochemical properties of the experimental sites before planting in 2014

The soil of the experimental site during the planting seasons was highly acidic. Both sites had low $\mathrm{pH}: \mathrm{H}_{2} \mathrm{O}$ and KCL (4.7, 3.8 respectively for early planting season and 4.3, 3.7 respectively for late planting season). It was also characterized with low organic matter, cat ion exchange capacity (CEC) and exchangeable bases (Table 1).

\section{Incidence of Zonocerus variegatus}

Cassava varieties differed significantly $(\mathrm{p}<0.05)$ in their susceptibility to $Z$. varigatus attack in both seasons (Table $2)$. At 4 months after planting (MAP), the incidence $(0.42 \%)$ of the pest on variety TMS 0510 was significantly higher than the incidence of the pest on other varieties but similar to that on variety TMS 01-1368 for the early season crops. At $6 \mathrm{MAP}$, incidence $(0.53 \%$ and $0.25 \%)$ of the insect on variety TMS 0510 during the seasons was significantly higher than that on other varieties, although statistically similar (0.35\%) with variety TMS 98-05-05 for early and variety TMS 01-1368 (0.22\%) for late season crops.

In the first six months, after planting fertilizer rates did not significantly $(p>0.05)$ influence the incidence of $Z$. variegatus on the field. At two MAP, the incidence of $\mathrm{Z}$. variegatus was generally low for late season crops. Conversely, the incidence $(0.50 \%)$ of the insect was increased during the early planting through the application of $200 \mathrm{~kg} \mathrm{NPK} \mathrm{ha}^{-1}$ at six MAP.

Mode of NPK fertilizer application had no significant $(\mathrm{p}$ $>0.05$ ) influence on the incidence of $Z$. variegatus in both planting seasons. However, split application of fertilizer gave the highest incidence $(0.42 \%)$ of $Z$. variegatus at 5 and 6 MAP of the early season.
There was an interaction between varieties $\mathrm{x}$ NPK fertilizer rates on $Z$. variegatus incidence at $6 \mathrm{MAP}$ of early season (Table 2). Application of $200 \mathrm{~kg} \mathrm{NPK} \mathrm{ha}^{-1}$ on variety TMS 98-05-05 gave a significantly higher incidence (1.00\%) of $Z$. variegatus than other rates, while application of $0 \mathrm{~kg}$ NPK ha ${ }^{-1}$ on variety TMS 419 and variety TMS 98-05-05 respectively, gave the least Z. variegatus incidence ( Table 3).

The incidence of $Z$. variegatus on cassava showed significant $(\mathrm{p}<0.05)$ difference among cassava varieties in late planting season (Table 4). At 9 MAP, incidence $(10.97 \%)$ of $Z$. variegatus on the variety TMS 98-05-05 was significantly higher than that on other varieties but similar $(10.65 \%)$ to that on the variety TMS 01-1368. Incidence $(7.56,7.50$ and $2.58 \%)$ of the insect on variety TMS 98-0505 was significantly higher than that of other varieties at 10 , 11, 12 MAP but similar to varieties TMS 01-1368 and TME 419, (6.94, 1.64 and 6.61, 2.50\%), respectively.

Fertilizer rates increased the incidence of $Z$. variegatus significantly $(p<0.05)$ in early season. At 10,11 and 12 MAP, application of $400 \mathrm{~kg}$ NPK ha ${ }^{-1}$, the incidence Zonocerus significantly increased (3.19, 3.44 and 6.67\%) compared to other rates; although not significantly higher than at $200 \mathrm{~kg}$ and $600 \mathrm{~kg} \mathrm{NPK} \mathrm{ha}^{-1}(2.61 \%, 3.00 \%$ and $3.14 \%, 2.72 \%$ respectively).

Mode of fertilizer application had no significant ( $\mathrm{p}>$ 0.05 ) influence on the incidence of $Z$. variegatus in both seasons. However, split-split method of fertilizer application gave the highest incidence of $Z$. variegatus $(9.10 \%)$ at 9 MAP of late planting.

There was no significant $(\mathrm{p}>0.05)$ interaction of cassava variety $x$ rate of NPK $x$ mode of fertilizer application (treatments) on the incidence of $Z$. variegatus on the field in both planting seasons (Table 4).

Table 1. Physical and chemical properties of the soil of the experimental sites

\begin{tabular}{|c|c|c|}
\hline Properties & Early season site & Late season site \\
\hline \multicolumn{3}{|l|}{ Particle size distribution (\%) } \\
\hline Clay & 10 & 14 \\
\hline Silt & 5 & 7 \\
\hline Fine sand & 33 & 43 \\
\hline Coarse sand & 52 & 36 \\
\hline Textural class & Loamy Sandy & Sandy Loam \\
\hline \multicolumn{3}{|l|}{ Chemical properties } \\
\hline $\mathrm{pH}\left(\mathrm{H}_{2} \mathrm{O}\right)$ & 4.7 & 4.8 \\
\hline $\mathrm{pH}(\mathrm{KCL})$ & 3.8 & 3.7 \\
\hline Organic carbon (\%) & 0.95 & 1.25 \\
\hline Organic matter $(\%)$ & 1.63 & 2.15 \\
\hline Total nitrogen (\%) & 0.084 & 0.098 \\
\hline \multicolumn{3}{|l|}{ Exchangeable bases (Meq/100 g Soil ) } \\
\hline Sodium $\left(\mathrm{Na}^{+}\right)$ & 0.12 & 0.15 \\
\hline Calcium $\left(\mathrm{Ca}^{+}\right)$ & 0.15 & 0.19 \\
\hline Magnesium $\left(\mathrm{Mg}^{+}\right)$ & 11.4 & 13.2 \\
\hline Cation exchange capacity (Meq/100 g Soil) & 0.4 & 2.4 \\
\hline Base Saturation & 13 & 16.4 \\
\hline Phosphorus (ppm) & 92.85 & 97.2 \\
\hline Exchangeable acidity(Meq/100 g Soil) & 6.53 & 3.73 \\
\hline Aluminum oxide $\left(\mathrm{AL}^{+}\right)$ & - & - \\
\hline Hydrogen oxide $\left(\mathrm{H}^{+}\right)$ & 2.4 & 2.4 \\
\hline
\end{tabular}


262

Table 2. Effect of varieties, rate, mode of NPK fertilizer and their interactions on the incidence of $Z$. variegatus on the field

\begin{tabular}{|c|c|c|c|c|c|c|c|c|c|c|}
\hline \multirow{2}{*}{ Treatments } & \multicolumn{5}{|c|}{ Early planting season } & \multicolumn{5}{|c|}{ Late planting season } \\
\hline & 2MAP & 3MAP & 4MAP & 5MAP & 6MAP & 2MAP & 3MAP & 4MAP & 5MAP & 6MAP \\
\hline \multicolumn{11}{|l|}{ Varieties } \\
\hline $\mathrm{TM}_{1}$ & $0.03(0.78)$ & $0.08(0.92)$ & $0.25(1.27)$ & $0.11(0.99)$ & $0.22(1.24)$ & $0.00(0.71)$ & $0.36(1.62)$ & $0.14(1.06)$ & $0.06(0.85)$ & $0.22(1.27)$ \\
\hline $\mathrm{TM}_{2}$ & $0.08(0.92)$ & $0.03(0.78)$ & $0.17(1.09)$ & $0.22(1.24)$ & $0.22(1.24)$ & $0.00(0.71)$ & $0.22(1.27)$ & $0.11(0.99)$ & $0.03(0.78)$ & $0.06(0.85)$ \\
\hline $\mathrm{TM}_{3}$ & $0.14(1.02)$ & $0.11(0.95)$ & $0.11(0.99)$ & $0.25(1.27)$ & $0.39(1.52)$ & $0.03(0.78)$ & $0.17(1.13)$ & $0.11(0.99)$ & $0.06(0.85)$ & $0.06(0.85)$ \\
\hline $\mathrm{TM}_{4}$ & $0.08(0.92)$ & $0.19(1.17)$ & $0.42(1.69)$ & $0.25(1.24)$ & $0.53(1.94)$ & $0.00(0.71)$ & $0.19(1.20)$ & $0.14(1.06)$ & $0.06(0.85)$ & $0.25(1.34)$ \\
\hline Mean & 0.91 & 0.95 & 1.26 & 1.18 & 1.48 & 0.72 & 1.31 & 1.02 & 0.83 & 1.08 \\
\hline $\operatorname{LSD}(0.05)$ & NS & NS & 0.50 & NS & 0.54 & NS & NS & NS & NS & 0.42 \\
\hline \multicolumn{11}{|l|}{$\begin{array}{c}\text { Rates } \\
(\mathrm{kg} / \mathrm{ha})\end{array}$} \\
\hline 0 & $0.08(0.88)$ & $0.11(0.95)$ & $0.28(1.34)$ & $0.22(1.20)$ & $0.28(1.34)$ & $0.00(0.71)$ & $0.22(1.27)$ & $0.08(0.92)$ & $0.00(0.71)$ & $0.17(1.13)$ \\
\hline 200 & $0.14(1.06)$ & $0.17(1.10)$ & $0.22(1.24)$ & $0.22(1.20)$ & $0.50(1.84)$ & $0.03(0.78)$ & $0.25(1.34)$ & $0.22(1.27)$ & $0.08(0.92)$ & $0.14(1.06)$ \\
\hline 400 & $0.06(0.85)$ & $0.06(0.85)$ & $0.22(1.24)$ & $0.25(1.27)$ & $0.25(1.31)$ & $0.00(0.71)$ & $0.28(1.41)$ & $0.08(0.92)$ & $0.06(0.85)$ & $0.11(0.99)$ \\
\hline 600 & $0.06(0.85)$ & $0.06(0.92)$ & $0.22(1.24)$ & $0.14(1.06)$ & $0.33(1.45)$ & $0.00(0.71)$ & $0.19(1.20)$ & $0.11(0.99)$ & $0.06(0.85)$ & $0.14(1.13)$ \\
\hline Mean & 0.91 & 0.95 & 1.26 & 1.18 & 1.48 & 0.72 & 1.31 & 1.02 & 0.83 & 1.08 \\
\hline $\operatorname{LSD}(0.05)$ & NS & NS & NS & NS & NS & NS & NS & NS & NS & NS \\
\hline \multicolumn{11}{|l|}{ Modes } \\
\hline Single & $0.06(0.87)$ & $0.06(0.87)$ & $0.21(1.21)$ & $0.29(1.40)$ & $0.29(1.39)$ & $0.02(0.76)$ & $0.29(1.45)$ & $0.13(1.08)$ & $0.042(0.81)$ & $0.10(0.97)$ \\
\hline Split & $0.08(0.89)$ & $0.21(1.18)$ & $0.31(1.45)$ & $0.42(1.63))$ & $0.42(1.63)$ & $0.00(0.71)$ & $0.17(1.34)$ & $0.10(1.02)$ & $0.062(0.87)$ & $0.10(0.97)$ \\
\hline Split-split & $0.10(0.97)$ & $0.04(0.81)$ & $0.19(1.13)$ & $0.31(1.42)$ & $0.31(1.42)$ & $0.00(0.71)$ & $0.25(1.13)$ & $0.15(0.97)$ & $0.042(0.81)$ & $0.23(1.29)$ \\
\hline Mean & 0.91 & 0.95 & 1.26 & 1.18 & 1.48 & 0.72 & 1.31 & 1.02 & 0.83 & 1.08 \\
\hline $\operatorname{LSD}(0.05)$ & NS & NS & NS & NS & NS & NS & NS & NS & NS & NS \\
\hline \multicolumn{11}{|l|}{ Interaction } \\
\hline VXR & NS & NS & NS & NS & 1.07 & NS & NS & NS & NS & NS \\
\hline VXM & NS & NS & NS & NS & NS & NS & NS & NS & NS & NS \\
\hline RXM & NS & NS & NS & NS & NS & NS & NS & NS & NS & NS \\
\hline VXRXM & NS & NS & NS & NS & NS & NS & NS & NS & NS & NS \\
\hline
\end{tabular}

Note: figures in parentheses are square root transformed values to which LSD is applicable, TM ${ }_{1}$-TMS 01-1368, TM2-TME 419,TM3-TMS 98-05-05,TM4-TMS 0510, MAP-month after planting, $\mathrm{V}=$ variety, $\mathrm{R}=$ rate, $\mathrm{M}=$ mate, Early planting season-May planting, NS-non significant, Late plant season-July planting.

Table 3. Interaction effect of varieties and NPK fertilizer rates on the incidence of $Z$. variegatus on the field at 6MAP

\begin{tabular}{|c|c|c|c|c|c|c|c|c|}
\hline \multirow{3}{*}{ Treatments } & \multicolumn{4}{|c|}{ Early planting season } & \multicolumn{4}{|c|}{ Late planting season } \\
\hline & \multicolumn{4}{|c|}{ Varieties } & \multicolumn{4}{|c|}{ Varieties } \\
\hline & $\mathrm{TM}_{1}$ & $\mathrm{TM}_{2}$ & $\mathrm{TM}_{3}$ & $\mathrm{TM}_{4}$ & $\mathrm{TM}_{1}$ & $\mathrm{TM}_{2}$ & $\mathrm{TM}_{3}$ & $\mathrm{TM}_{4}$ \\
\hline \multicolumn{9}{|l|}{ Rates(kg/ha) } \\
\hline 0 & $0.44(1.70)$ & $0.33(1.42)$ & $0.11(0.99)$ & $0.22(1.27)$ & $0.22(1.27)$ & $0.00(0.71)$ & $0.00(0.71)$ & $0.33(1.55)$ \\
\hline 200 & $0.22(1.27)$ & $0.22(1.27)$ & $1.00(2.69)$ & $0.56(2.11)$ & $0.111(0.99)$ & $0.11(0.99)$ & $0.11(0.99)$ & $0.00(0.71)$ \\
\hline 400 & $0.11(0.99)$ & $0.00(0.71)$ & $0.00(0.71)$ & $0.89(2.82)$ & $0.22(1.27)$ & $0.22(1.27)$ & $0.22(1.27)$ & $0.33(1.55)$ \\
\hline 600 & $0.11(0.99)$ & $0.33(1.55)$ & $0.44(1.70)$ & $0.44(1.56)$ & $0.33(1.55)$ & $0.00(0.71)$ & $0.22(1.27)$ & $0.44(1.83)$ \\
\hline Mean & 1.48 & 1.48 & 1.48 & 1.48 & 1.08 & 1.08 & 1.08 & 1.08 \\
\hline $\operatorname{LSD}(0.05)$ & & 1.07 & & & & NS & & \\
\hline
\end{tabular}

Note: figures in parentheses are square root transformed values to which LSD is applicable,TM1-TMS 01-1368, TM2-TME 419TM3-TMS 98-05-05, TM4-TMS 0510,MAP-month after planting, Early planting season-May planting, ns-non-significant Late planting season-July planting

\section{Incidence of termites}

The incidence of termites on cassava showed significant $(\mathrm{p}<0.05)$ difference among varieties in late season (Table 5). At 2, 3, 4, 5 and $6 \mathrm{MAP}$, incidence of termites on variety TMS 0510 (3.06, 6.19, 3.31 and 5.39\% respectively), were significantly higher compared to that on other varieties, while the incidence on variety TMS 01-1368 (0.31, 0.97, 0.42 and $0.78 \%$ respectively) were the least.

Rate and mode of fertilizer application did not significantly $(p>0.05)$ influence the incidence of termites in both seasons, all through the sampling period.

There were interaction effects of varieties $\mathrm{x}$ mode of NPK fertilizer application on incidence of termites on the field at 2 MAP of early and 4 MAP of late seasons respectively (Table 5). Single application mode of fertilizer on variety TMS 01-1368 gave significantly higher incidence $(2.75 \%)$ of termites than other rates (Table 6), though the effect was mainly from the variety.

The incidence of termites on cassava showed significant $(p<0.05)$ difference among varieties in late planting seasons (Table 7). At 7, 8, 9, 10, 11 and 12 MAP, the incidence $(6.06,5.67,6.61,7.28,7.00$ and $6.39 \%)$ of the pest on variety TMS 0510 was significantly high compared to that on other varieties.

There was no significant $(\mathrm{p}>0.05)$ interaction of cassava variety $x$ rate of NPK $x$ mode of fertilizer application on the incidence of termites on the field in both planting seasons (Table 7). 
Table 4. Effect of varieties, rate, mode of NPK fertilizer and their interactions on the incidence of $Z$. variegatus on the field

\begin{tabular}{|c|c|c|c|c|c|c|c|c|c|c|c|c|}
\hline \multirow{2}{*}{ Treatments } & \multicolumn{6}{|c|}{ Early planting season } & \multicolumn{6}{|c|}{ Late planting season } \\
\hline & $7 \mathrm{MAP}$ & $8 \mathrm{MAP}$ & 9MAP & 10MAP & 11MAP & 12MAP & $7 \mathrm{MAP}$ & $8 \mathrm{MAP}$ & 9MAP & 10MAP & 11MAP & 12MAP \\
\hline \multicolumn{13}{|l|}{ Varieties } \\
\hline $\mathrm{TM}_{1}$ & $0.25(1.31)$ & $0.06(0.85)$ & $0.19(1.13)$ & $2.58(4.53)$ & $2.69(4.55)$ & $5.14(6.85)$ & $0.22(1.27)$ & $1.50(3.14)$ & $10.64(9.80)$ & $6.69(7.61)$ & $6.94(8.15)$ & $1.64(3.86)$ \\
\hline $\mathrm{TM}_{2}$ & $0.25(1.34)$ & $0.08(0.92)$ & $0.22(1.27)$ & $2.97(4.95)$ & $2.69(4.61)$ & $5.36(7.01)$ & $0.08(0.92)$ & $1.03(2.48)$ & $8.44(8.89)$ & $5.83(7.38)$ & $6.61(8.06)$ & $2.50(4.68)$ \\
\hline $\mathrm{TM}_{3}$ & $0.50(1.84)$ & $0.14(1.06)$ & $0.22(1.24)$ & $2.47(4.68)$ & $2.64(4.91)$ & $5.25(7.06)$ & $0.14(1.06)$ & $1.25(2.62)$ & $10.97(10.21)$ & $7.56(8.39)$ & $7.50(8.49)$ & $2.58(4.78)$ \\
\hline $\mathrm{TM}_{4}$ & $0.39(1.59)$ & $0.11(0.99)$ & $0.22(1.24)$ & $2.67(4.71)$ & $3.17(5.27)$ & $6.17(7.55)$ & $0.28(14.00)$ & $1.08(2.73)$ & $3.56(5.41)$ & $2.39(4.43)$ & $3.00(4.96)$ & $1.36(2.81)$ \\
\hline Mean & 1.52 & 0.95 & 1.22 & 4.72 & 4.84 & 7.12 & 1.17 & 2.74 & 8.57 & 6.95 & 7.41 & 4.03 \\
\hline $\operatorname{LSD}(0.05)$ & NS & NS & NS & NS & NS & NS & NS & NS & 1.32 & 1.11 & 0.95 & 0.95 \\
\hline \multicolumn{13}{|l|}{$\begin{array}{c}\text { Rates } \\
(\mathrm{kg} / \mathrm{ha})\end{array}$} \\
\hline 0 & $0.36(1.59)$ & $0.11(0.99)$ & $0.14(1.02)$ & $1.76(3.61)$ & $2.03(4.15)$ & $4.19(6.30)$ & $0.14(1.06)$ & $0.89(2.32)$ & $7.64(8.00)$ & $4.78(6.50)$ & $5.47(7.08)$ & $2.03(4.10)$ \\
\hline 200 & $0.39(1.59)$ & $0.08(0.92)$ & $0.28(1.38)$ & $3.14(5.27)$ & $2.72(4.62)$ & $5.08(6.86)$ & $0.08(0.92)$ & $1.17(2.35)$ & $7.94(8.32)$ & $5.78(7.17)$ & $5.83(7.42)$ & $1.89(3.88)$ \\
\hline 400 & $0.25(1.31)$ & $0.03(0.78)$ & $0.22(1.24)$ & $3.19(5.35)$ & $3.44(5.50)$ & $6.67(7.92)$ & $0.25(1.34)$ & $1.17(3.00)$ & $9.56(9.21)$ & $6.19(7.09)$ & $6.56(7.74)$ & $1.89(3.83)$ \\
\hline 600 & $0.39(1.59)$ & $0.17(1.129)$ & $0.22(1.24)$ & $2.61(4.64)$ & $3.00(5.08)$ & $5.97(7.39)$ & $0.25(1.34)$ & $1.50(3.31)$ & $8.47(8.77)$ & $5.72(7.04)$ & $6.19(7.41)$ & $2.28(4.32)$ \\
\hline Mean & 1.52 & 0.95 & 1.22 & 4.72 & 4.84 & 7.12 & 1.17 & 2.74 & 8.57 & 6.95 & 7.41 & 4.03 \\
\hline $\operatorname{LSD}(0.05)$ & NS & NS & NS & 0.96 & 0.94 & 0.89 & NS & NS & NS & NS & NS & NS \\
\hline \multicolumn{13}{|l|}{ Modes } \\
\hline Single & $0.38(1.55)$ & $0.10(0.97)$ & $0.25(1.34)$ & $2.88(4.85)$ & $3.25(5.35)$ & $6.27(7.57)$ & $0.15(1.08)$ & $1.12(2.56)$ & $8.67(8.65)$ & $6.02(7.15)$ & $6.46(7.68)$ & $2.10(4.06)$ \\
\hline Split & $0.39(1.63)$ & $0.10(0.97)$ & $0.22(1.21)$ & $2.73(4.81)$ & $2.60(4.62)$ & $5.00(6.86)$ & $0.21(1.24)$ & $1.29(2.77)$ & $7.44(8.18)$ & $5.06(6.75)$ & $5.40(7.04)$ & $1.92(3.88)$ \\
\hline Split-split & $0.27(1.37)$ & $0.08(0.92)$ & $0.17(1.10)$ & $2.88(4.50)$ & $2.54(4.54)$ & $5.17(6.91)$ & $0.19(1.18)$ & $1.23(2.90)$ & $9.10(8.89)$ & $5.77(6.75)$ & $6.19(7.51)$ & $2.04(4.15)$ \\
\hline Mean & 1.52 & 0.95 & 1.22 & 4.72 & 4.84 & 7.12 & 1.17 & 2.74 & 8.57 & 6.95 & 7.41 & 4.03 \\
\hline $\operatorname{LSD}(0.05)$ & NS & NS & NS & NS & NS & NS & NS & NS & NS & NS & NS & NS \\
\hline \multicolumn{13}{|l|}{ Interaction } \\
\hline VXR & NS & NS & NS & NS & NS & NS & NS & NS & NS & NS & NS & NS \\
\hline VXM & NS & NS & NS & NS & NS & NS & NS & NS & NS & NS & NS & NS \\
\hline RXM & NS & NS & NS & NS & NS & NS & NS & NS & NS & NS & NS & NS \\
\hline VXRXM & NS & NS & NS & NS & NS & NS & NS & NS & NS & NS & NS & NS \\
\hline
\end{tabular}

Note: figures in parentheses are square root transformed values to which LSD is applicable,TM1-TMS 01-1368, TM2-TME 419, TM3-TMS 98-05-05,TM4-

TMS0510, MAP- month after planting, $\mathrm{V}=$ variety, $\mathrm{R}=$ rate, $\mathrm{M}=$ mate,Early planting season-May planting, Late planting season-July planting, NS-non significant

Table 5. Effect of varieties, rate, mode of NPK fertilizer and their interactions on the incidence of termites on the field

\begin{tabular}{|c|c|c|c|c|c|c|c|c|c|c|}
\hline \multirow{2}{*}{ Treatments } & \multicolumn{5}{|c|}{ Early planting season } & \multicolumn{5}{|c|}{ Late planting season } \\
\hline & 2MAP & $3 \mathrm{MAP}$ & $4 \mathrm{MAP}$ & $5 \mathrm{MAP}$ & 6MAP & $2 \mathrm{MAP}$ & 3MAP & $4 \mathrm{MAP}$ & $5 \mathrm{MAP}$ & $6 \mathrm{MAP}$ \\
\hline \multicolumn{11}{|l|}{ Varieties } \\
\hline $\mathrm{TM}_{1}$ & $1.69(3.66)$ & $1.53(3.15)$ & $1.53(3.15)$ & $1.67(3.36)$ & $1.78(3.58)$ & $0.31(1.37)$ & $0.31(1.37)$ & $0.97(2.61)$ & $0.42(1.69)$ & $0.78(2.25)$ \\
\hline $\mathrm{TM}_{2}$ & $1.39(3.29)$ & $0.83(2.44)$ & $0.83(2.44)$ & $1.17(2.90)$ & $1.17(2.90)$ & $1.11(2.93)$ & $1.11(2.93)$ & $2.00(4.06)$ & $0.75(2.24)$ & $1.53(3.62)$ \\
\hline $\mathrm{TM}_{3}$ & $1.44(3.28)$ & $1.22(2.82)$ & $1.22(2.82)$ & $1.67(3.54)$ & $1.72(3.59)$ & $2.06(4.26)$ & $1.94(4.05)$ & $3.61(5.87)$ & $1.83(3.90)$ & $2.69(5.02)$ \\
\hline $\mathrm{TM}_{4}$ & $1.25(2.91)$ & $1.28(2.89)$ & $1.25(2.86)$ & $1.50(3.19)$ & $1.56(3.33)$ & $3.17(5.38)$ & $3.06(5.22)$ & $6.19(7.74)$ & $3.31(5.56)$ & $5.39(7.26)$ \\
\hline Mean & 3.29 & 2.83 & 2.82 & 3.25 & 3.35 & 3.49 & 3.39 & 5.07 & 3.35 & 4.54 \\
\hline $\operatorname{LSD}(0.05)$ & NS & NS & NS & NS & NS & 0.74 & 0.80 & 0.82 & 0.86 & 0.79 \\
\hline 0 & $1.44(3.27)$ & $1.31(2.95)$ & $1.28(2.88)$ & $1.64(3.30)$ & $1.72(3.51)$ & $1.56(3.40)$ & $1.67(3.55)$ & $3.42(5.49)$ & $1.50(3.19)$ & $2.47(4.51)$ \\
\hline 200 & $1.61(3.61)$ & $1.17(2.82)$ & $1.19(2.89)$ & $1.50(3.38)$ & $1.58(3.50)$ & $1.56(3.41)$ & $1.28(3.04)$ & $2.89(4.57)$ & $1.64(3.33)$ & $2.47(4.27)$ \\
\hline 400 & $1.14(2.81)$ & $1.14(2.65)$ & $1.14(2.65)$ & $1.33(3.04)$ & $1.36(3.07)$ & $1.64(3.35)$ & $1.58(3.21)$ & $3.19(5.17)$ & $1.61(3.49)$ & $2.75(4.76)$ \\
\hline 600 & $1.58(3.45)$ & $1.25(2.89)$ & $1.22(2.85)$ & $1.53(3.29)$ & $1.56(3.32)$ & $1.89(3.79)$ & $1.89(3.79)$ & $3.28(5.05)$ & $1.56(3.39)$ & $2.69(4.62)$ \\
\hline Mean & 3.29 & 2.83 & 2.82 & 3.25 & 3.35 & 3.49 & 3.39 & 5.07 & 3.35 & 4.54 \\
\hline $\operatorname{LSD}(0.05)$ & NS & NS & NS & NS & NS & NS & NS & NS & NS & NS \\
\hline Single & $1.54(3.39)$ & $1.25(2.83)$ & $1.27(2.88)$ & $1.67(3.47)$ & $1.71(3.52)$ & $1.63(3.38)$ & $1.58(3.26)$ & $3.33(5.17)$ & $1.58(3.32)$ & $2.67(4.63)$ \\
\hline Split & $1.46(3.21)$ & $1.19(2.78)$ & $1.19(2.78)$ & $1.46(3.23)$ & $1.52(3.36)$ & $1.92(3.86)$ & $1.79(3.70)$ & $3.35(5.20)$ & $1.58(3.31)$ & $2.73(4.63)$ \\
\hline Split-split & $1.33(3.26)$ & $1.21(2.87)$ & $1.17(2.79)$ & $1.38(3.04)$ & $1.44(3.17)$ & $1.44(3.22)$ & $1.44(3.22)$ & $2.90(4.85)$ & $1.56(3.42)$ & $2.40(4.36)$ \\
\hline Mean & 3.29 & 2.83 & 2.82 & 3.25 & 3.35 & 3.49 & 3.39 & 5.07 & 3.35 & 4.54 \\
\hline $\operatorname{LSD}(0.05)$ & NS & NS & NS & NS & NS & NS & NS & NS & NS & NS \\
\hline \multicolumn{11}{|l|}{ Interaction } \\
\hline VXR & NS & NS & NS & NS & NS & NS & NS & 1.64 & NS & NS \\
\hline VXM & 1.59 & NS & NS & NS & NS & NS & NS & NS & NS & NS \\
\hline RXM & 1.59 & NS & NS & NS & NS & NS & NS & NS & NS & NS \\
\hline VXRXM & NS & NS & NS & NS & NS & NS & NS & NS & NS & NS \\
\hline
\end{tabular}

Note: figures in parentheses are square root transformed values to which LSD is applicable, TM1-TMS 01-1368, TM2-TME 419,TM3- TMS 98-05-05, TM4-TMS 0510,MAP-month after planting, $V=$ variety, $\mathrm{R}=$ rate, $\mathrm{M}=$ mate, Early planting season-May planting, NS-non significant, Late planting season-July planting 
Table 6. Interaction effect of varieties and mode of NPK fertilizer on the incidence of termites on the field at 2MAP

\begin{tabular}{|c|c|c|c|c|c|c|c|c|}
\hline \multirow[b]{3}{*}{ Treatments } & \multicolumn{4}{|c|}{ Early planting season } & \multicolumn{4}{|c|}{ Late planting season } \\
\hline & \multicolumn{4}{|c|}{ Varieties } & \multicolumn{4}{|c|}{ Varieties } \\
\hline & $\mathrm{TM}_{1}$ & $\mathrm{TM}_{2}$ & $\mathrm{TM}_{3}$ & $\mathrm{TM}_{4}$ & $\mathrm{TM}_{1}$ & $\mathrm{TM}_{2}$ & $\mathrm{TM}_{3}$ & $\mathrm{TM}_{4}$ \\
\hline \multicolumn{9}{|l|}{ Mode } \\
\hline Single & $2.75(4.95)$ & $1.58(3.72)$ & $1.08(2.77)$ & $0.75(2.13)$ & $0.25(1.34)$ & $0.83(2.24)$ & $1.92(4.19)$ & $3.50(5.73)$ \\
\hline Split & $1.25(3.04)$ & $1.33(2.93)$ & $1.42(3.29)$ & $1.83(3.58)$ & $0.67(2.06)$ & $1.25(3.23)$ & $2.58(4.63)$ & $3.17(5.51)$ \\
\hline Split-split & $1.08(2.99)$ & $1.25(3.23)$ & $1.83(3.79)$ & $1.17(3.02)$ & $0.00(0.71)$ & $1.25(3.33)$ & $1.67(3.95)$ & $2.83(4.91)$ \\
\hline Mean & 3.29 & 3.29 & 3.29 & 3.29 & 3.49 & 3.49 & 3.49 & 3.49 \\
\hline $\operatorname{LSD}(0.05)$ & & 1.59 & & & & NS & & \\
\hline
\end{tabular}

Note: figures in parentheses are square root transformed values to which LSD is applicable, TM1-TMS 01-1368, TM2-TME 419,TM3-TMS 98-05-05, TM4-TMS 0510,MAP-month after planting, Early planting season-May planting, Late planting season-July planting, NS-non significant

Table 7. Effect of varieties, rate, mode of NPK fertilizer and their interactions on the incidence of termites on the field

\begin{tabular}{|c|c|c|c|c|c|c|c|c|c|c|c|c|}
\hline \multirow{2}{*}{ Treatments } & \multicolumn{6}{|c|}{ Early planting season } & \multicolumn{6}{|c|}{ Late planting season } \\
\hline & $7 \mathrm{MAP}$ & $8 \mathrm{MAP}$ & 9MAP & 10MAP & 11MAP & 12MAP & $7 \mathrm{MAP}$ & $8 \mathrm{MAP}$ & 9MAP & 10MAP & 11MAP & 12MAP \\
\hline \multicolumn{13}{|l|}{ Varieties } \\
\hline $\mathrm{TM}_{1}$ & $2.58(4.77)$ & $2.83(5.01)$ & $3.47(5.61)$ & $3.53(5.66)$ & $3.53(5.66)$ & $4.19(6.19)$ & $1.44(3.61)$ & $1.53(3.68)$ & $1.53(3.70)$ & $2.28(4.54)$ & $2.33(4.60)$ & $3.11(5.17)$ \\
\hline $\mathrm{TM}_{2}$ & $1.83(4.11)$ & $2.03(4.31)$ & $2.67(4.99)$ & $2.72(5.04)$ & $2.72(5.04)$ & $3.39(5.61)$ & $2.14(4.46)$ & $2.42(4.72)$ & $2.47(4.76)$ & $3.14(5.38)$ & $3.14(5.38)$ & $3.08(5.34)$ \\
\hline $\mathrm{TM}_{3}$ & $2.44(4.67)$ & $2.64(4.88)$ & $3.31(5.50)$ & $3.39(5.57)$ & $3.39(5.57)$ & $4.06(6.12)$ & $3.31(5.53)$ & $3.78(5.91)$ & $3.92(6.05)$ & $4.58(6.53)$ & $4.58(6.53)$ & $4.53(6.49)$ \\
\hline $\mathrm{TM}_{4}$ & $2.22(4.38)$ & $2.39(4.57)$ & $3.06(5.19)$ & $3.06(5.25)$ & $3.06(5.25)$ & $3.72(5.78)$ & $6.06(7.69)$ & $5.67(7.47)$ & $6.61(8.10)$ & $7.28(8.48)$ & $7.00(8.22)$ & $6.39(7.69)$ \\
\hline Mean & 4.48 & 4.7 & 5.32 & 5.38 & 5.38 & 5.92 & 5.32 & 5.44 & 5.65 & 6.23 & 6.18 & 6.17 \\
\hline $\operatorname{LSD}(0.05)$ & NS & NS & NS & NS & NS & NS & 0.67 & 0.73 & 0.70 & 0.65 & 0.75 & 0.88 \\
\hline \multicolumn{13}{|l|}{ Rates $(\mathrm{kg} / \mathrm{ha})$} \\
\hline 0 & $2.44(4.52)$ & $2.69(4.79)$ & $3.33(5.35)$ & $3.42(5.47)$ & $3.42(5.47)$ & $4.08(6.00)$ & $3.06(5.18)$ & $3.14(5.31)$ & $3.67(5.71)$ & $4.42(6.34)$ & $4.17(6.11)$ & $4.31(6.25)$ \\
\hline 200 & $2.31(4.57)$ & $2.67(4.93)$ & $3.28(5.49)$ & $3.39(5.57)$ & $3.39(5.57)$ & $4.06(6.07)$ & $3.17(5.18)$ & $3.22(5.25)$ & $3.44(5.39)$ & $4.11(5.96)$ & $4.14(5.99)$ & $4.33(6.13)$ \\
\hline 400 & $2.06(4.31)$ & $2.17(4.44)$ & $2.83(5.14)$ & $2.94(5.25)$ & $2.94(5.25)$ & $3.61(5.82)$ & $3.39(5.59)$ & $3.69(5.86)$ & $3.81(5.94)$ & $4.47(6.53)$ & $4.47(6.53)$ & $4.42(6.43)$ \\
\hline 600 & $2.28(4.53)$ & $2.36(4.62)$ & $3.06(5.32)$ & $2.94(5.24)$ & $2.94(5.24)$ & $3.61(5.79)$ & $3.33(5.34)$ & $3.33(5.37)$ & $3.61(5.57)$ & $4.28(6.10)$ & $4.28(6.10)$ & $4.06(5.89)$ \\
\hline Mean & 4.48 & 4.70 & 5.32 & 5.38 & 5.38 & 5.92 & 5.32 & 5.44 & 5.65 & 6.23 & 6.18 & 6.17 \\
\hline $\operatorname{LSD}(0.05)$ & NS & NS & NS & NS & NS & NS & NS & NS & NS & NS & NS & NS \\
\hline \multicolumn{13}{|l|}{ Modes } \\
\hline Single & $2.46(4.63)$ & $2.69(4.87)$ & $3.29(5.42)$ & $3.25(5.39)$ & $3.25(5.39)$ & $3.92(5.93)$ & $3.25(5.30)$ & $3.27(5.39)$ & $3.71(5.70)$ & $4.38(6.25)$ & $4.19(6.07)$ & $4.52(6.37)$ \\
\hline Split & $2.23(4.42)$ & $2.42(4.62)$ & $3.08(5.24)$ & $3.15(5.33)$ & $3.15(5.33)$ & $3.81(5.86)$ & $3.40(5.45)$ & $3.54(5.57)$ & $3.77(5.76)$ & $4.50(6.38)$ & $4.44(6.33)$ & $4.15(6.06)$ \\
\hline Split-split & $2.12(4.40)$ & $2.31(4.61)$ & $3.00(5.31)$ & $3.12(5.42)$ & $3,12(5.42)$ & $3.92(5.97)$ & $3.06(5.22)$ & $3.23(5.36)$ & $3.42(5.49)$ & $4.08(6.07)$ & $4.17(6.15)$ & $4.17(6.09)$ \\
\hline Mean & 4.48 & 4.7 & 5.32 & 5.38 & 5.38 & 5.92 & 5.32 & 5.44 & 5.65 & 6.23 & 6.18 & 6.17 \\
\hline $\operatorname{LSD}(0.05)$ & NS & NS & NS & NS & NS & NS & NS & NS & NS & NS & NS & NS \\
\hline \multicolumn{13}{|l|}{ Interaction } \\
\hline VXR & NS & NS & NS & NS & NS & NS & NS & NS & NS & NS & NS & NS \\
\hline VXM & NS & NS & NS & NS & NS & NS & NS & NS & NS & NS & NS & NS \\
\hline RXM & NS & NS & NS & NS & NS & NS & NS & NS & NS & NS & NS & NS \\
\hline VXRXM & NS & NS & NS & NS & NS & NS & NS & NS & NS & NS & NS & NS \\
\hline
\end{tabular}

\section{Discussion}

The influence of NPK fertilizer application on the incidence of grasshopper (Zonocerus variegatus) and termite (Isoptera) was investigated on four cassava varieties. There was a considerable $Z$. variegatus attack on all the varieties, especially varieties TMS 05 10, TMS 01-1368 and TMS 9805-05 in both seasons. While variety TME 419 showed some levels of resistance to this pest considering the low incidence of the pest on it. This indicates the endemic and serious nature of this pest in the region, where its past outbreaks has been reported. Almost all the activities of this pest, from oviposition to feeding are centred on cassava fields (Page, 1978; Bellotti, 2002; Oku et al., 2011; Bamidele and Muse, 2012). Despite the presence of hydrogen cyanide in cassava $Z$. variegatus still attack the crop due to its ability to break the defence system. According to Agboola et al. (2013), the survival of $Z$. variegatus depends on the presence of the enzyme rhodanese, which shows high activity and has suitable kinetic properties in the gut of grasshopper that feeds mainly on cassava leaves, which are cyanogenic.

Termite attack spread to all the varieties, with variety 'TMS 05 10', being the most attacked. The nature of cassava stems could be responsible for variation of termite incidence among varieties. Cassava varieties in which the stem bark is thick and hard with a reduced diameter of marrow are more tolerant to the action of termites, while those with thin and fragile bark and large stem marrow are sensitive (Faye et al., 2015). The reason for less damage on other varieties is probably due to non-preference since termite as wood degrader has cellulolytic abilities for efficient wood degradation enabled by a special complement of gut symbionts (Honogoh, 2011).

Application of NPK fertilizer increased the incidence of $Z$. variegatus in all the plots, the magnitude of the increase being associated with levels of fertilization to insect attack. The significant more increase in $Z$. variegatus incidence in plots with fertilizer such as 400, 200 and $600 \mathrm{~kg} / \mathrm{ha} \mathrm{NPK}$ fertilizer application rates is notable, due to the vegetative growth the crop caused by fertilization, which reflected at the later part of the experiment when the crops could attract the pest. This confirms the findings by Chau et al. (2003) who reported that some studies have also been documented on how the shift from organic soil management to chemical fertilizers has increased the potential of certain insects and diseases to cause economic losses. Weedraddana and Evenden (2018) stated that fertilizer treatment and canola 
variety influenced oviposition behaviour of bertha armyworm and its larval performance, that moderate and high fertilizer treatments gave same number of eggs laid. Baidoo and Mochiah (2011) also stated that increased nutrient application to the soil enhances plant growth and thus improves the yield of crops. However, improved vegetative growth can make the plant more attractive to pests attack and destruction. The lack of any significant influence of NPK fertilizer on termite incidence is line with findings by Feller (1995) who reported that herbivory of dwarf red mangrove trees by two specialist herbivores of the tree increased with the application of $P$ and NPK fertilizers compared to $\mathrm{N}$-fertilizers and control trees, while fertilization had no effects on the feeding rates of generalist herbivores.

The absence of any significant influence of fertilizer mode on the incidence of both pests indicates that there was a masking effect of fertilizer rates on it. Even though split fertilizer application helps to optimize nutrient management and target plant needs (The fertilizer institute, 2015).

\section{Conclusions}

The abundance of $Z$. variegatus in cassava field could be influenced by fertilization and cassava variety while termite damage could be determined by cassava variety. It can form the basis for determining the endemic nature of this pest in an environment and help to predict future outbreak. This underscores the importance of proper evaluation of new cultivars in a place against pests that are of economic importance to the crop.

\section{Conflicts of interest}

The authors declare that there are no conflicts of interest related to this article.

\section{References}

Ademolu KO, Idowu BA, Oke AO (2011). Impact of reproductive activities on the tissues of (Zonocerus variegates) adult grasshopper (Orthoptera: Pygomorphidae).Florida Entomologist 94(4):993-997.

Agboola KF, Igue UB (2013). Detoxification of cyanide in insects.1 purification and some properties of rhodanese from the gut of the variegated grasshopper (Zonocerus variegatus) (Orthoptera: Pyrgorphidae). International Journal of Tropical Insect Science 33(3):153-162.

Amtmann A, Troufflard S, Armengaud P (2008). The effect of potassium nutrition on pest and disease resistance in plants. Physiologia Plantarum 133(4):682-691.

Baidoo PK, Mochiah MB (2011).The influence of nutrient application on pests natural enemies of pests okra Abelmoschus esculentus (L.) (Moench.).Journal of Applied Biological Science 1:2765-2771.

Baidoo PK, Mochiah MB, Asare D, Sefah AA (2018). Sustainable agricultural research. Canadian Center for Science and Education 7 (1).

Bamidele AO, Muse WA (2012). A morphometric study of the variegated grasshopper Zonocerus variegatus (I) (Othoptera: Pyromorphidae) from parts of southern Nigeria. Ife Journal of Science 14(1):61-73.
Bellotti AC (2002). Pest and Disease Management Project. Centro Internacional de Agricultura Tropical (ClA T).A.A. 6713. Cali.

Chau LM, Cheng J, Heong KL (2003). Impacts of nutrition management on insect pests and diseases of rice. Omonrice 11:93-102.

Dauda SN, Ajayi FA, Ndor E (2008). Growth and yield of watermelon (Citrullus lanatus) as affected by poultry manure application. Journal of Agricultural and Social Sciences 4(3):121-124.

Ewulo BS (2005). Effect of poultry dung and cattle manure on chemical and properties of clay and sandy clay loam soil. Journal of Animal and Veterinary Advances 4(10):839-841.

Faye A, Sall SY, Dieynaba K, Papa DM, Demba F, Sane D (2015). Characteristics of cassava (Manihot esculenta Crantz) stem on its varietal vulnerability to termites ravaging cuttings Odototermes sp. aff. erraticus. African Journal of Agricultural Research 10(19):2083-2088.

Feller IC (1995). Effects of nutrient enrichment on growth and herbivory of dwarf red mangrove (Rhizophora mangle). Ecological Monographs 65(4):477-505.

Hongoh Y (2011). Toward the functional analysis of uncultivable, symbiotic microorganisms in the termite gut. Cell and Molecular Life Sciences 68(8):1311-1325.

Ikpe FN, Powell JM (2002). Nutrient cycling practices and change in soil properties in livestock farming systems of western Niger Republic of West Africa. Nutrient Cyclingin Agroecosystems 62(1):37-45.

Mbagwu JSC (1992). Improving the productivity of a degraded ultisol in Nigeria using organic and inorganic amendments. Part 1: Chemical properties and maizeyield. Bioresource Technology 42(2):149-154.

NCO (2019). North Country Organics. Fertility and insects. https://norganics.com/index-2/technical-articles/fertility-and-insects/.

Obi IU (2002). Statistical methods of detecting differences between treatment means and research methodology issues in laboratory and field experiment. Nsukka, Nigeriapp 3444 .

Ogbalu OK (1999). The effects of different traditional sources of nutrients on the infestation of pepper fruits by the pepper fruit fly, Atherigona orientalis (schiner) in Nigeria. Journal Agronomy and Crop Science 182(1):65-71.

Ogedegbe AB, Ezeh AE (2015). Effect of variety and nutrient on insect pest infestation of Amaranthus spp. Journal of Applied Sciences and Environmental Management 19(2):251-256.

OkuEE, ArongGA, Bassey DA (2011).Species compostion of grasshoppers (Othoptera) in open plots and farmlands in Calabar Metropolis, southern Nigeria. Pakistan Journal of Biological Science 14(8):507-510.

Page WW (1978). The biology and control of grasshopper Zonocerus variegatus.Pans 24(3):270-277.

Weeraddana CS, Evenden ML (2018). Canola nutrition and variety effect on oviposition and offspring performance in the generalist herbivore, Manestra configurata (Lepidoptera: Noctuidae). Journal of Economic Entomology 11(4):1702-1710.

Yardim N, Erdal E, Clive A (2004). Effects of organic and synthetic fertilizer sources on pest and predatory insects associated with tomatoes. Phytoparasitica31(4):324329.

Youdeowei A (1974). Dissection of variegated grasshopper: Zonocerus variegatus (L). Oxford University Press, Ibadan, Nigeria. 